\title{
Risk of post-traumatic epilepsy after severe head injury in patients with at least one seizure
}

This article was published in the following Dove Press journal:

Neuropsychiatric Disease and Treatment

30 August 2017

Number of times this article has been viewed

\section{Wei Chen' \\ Ming-De $\mathrm{Li}^{2}$ \\ Gui-Fang Wang' \\ Xia-Feng Yang' \\ Lin Liu' \\ Fan-Gang Meng ${ }^{3}$}

'Department of Neurology, Liaocheng People's Hospital, Liaocheng,

${ }^{2}$ Department of Neurosurgery,

The People's Hospital in Ling

Cheng District, DeZhou, Shandong,

${ }^{3}$ Department of Functional

Neurosurgery, Beijing Neurosurgical

Institute, Capital Medical University,

Beijing, China

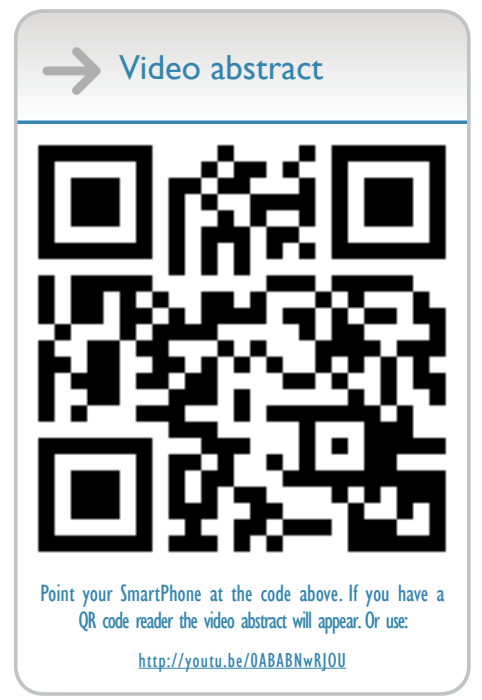

Correspondence: Fan-Gang Meng Department of Functional Neurosurgery, Beijing Neurosurgical Institute, Capital Medical University, No 6 Tiantan Xili, Dongcheng District, Beijing 100050, China

Tel +861067096767

Fax +861067057507

Email fgmeng@ccmu.edu.cn
Background: To explore the incidence and risk factors, including type of seizures for post-traumatic epilepsy (PTE) after severe traumatic brain injury (TBI).

Subjects and methods: This was a retrospective follow-up study of patients discharged from Liaocheng People's Hospital between March 2011 and June 2015 with a diagnosis of post-traumatic seizures. Risk factors for PTE were evaluated in 68 inpatients by using KaplanMeier curves and the Cox model.

Results: Complete clinical information was available for 68 patients. A total of 54 cases (79.4\%) were diagnosed as presenting with PTE, occurring from 10 days to 179 months after severe TBI. Nineteen out of 54 cases (35.2\%) had been defined as PTE within the first 6 months after the trauma, 17 cases (31.5\%) within 7-12 months, 8 cases (14.8\%) within 13-24 months, 2 cases (3.7\%) within 25-36 months, and 8 cases (14.8\%) within 37-179 months after the TBI. The Kaplan-Meier curves demonstrated that simple partial seizures, surgical treatment, and onset of seizures occurring within 6 months after injury were associated with PTE.

Conclusions: The Cox model indicated that, for patients aged $>34$ years at the time of injury, the PTE risk was 2.55 times greater than for those aged $\leq 34$ years. In addition, simple partial seizures, surgical treatment and onset of seizures occurring within 6 months after injury were significant risk factors for the development of PTE.

Keywords: post-traumatic epilepsy, risk factor, post-traumatic seizure

\section{Background}

It is clear that epilepsy is a frequent consequence of brain injury, even many years after the injury, accidents and medical complications commonly occur during seizures. In view of the adverse impact that post-traumatic epilepsy (PTE) can have on quality of life, questions concerning patients when seizures develop include: How frequent will they be? How to predict the risk factors of PTE efficiently and reduce its incidence? The answers to these questions have important implications for prevention. Unfortunately, the factors that predict the risk of PTE are still not well established. The frequency of seizures in patients with PTE is not associated with any identifiable variables and often varies widely, even within generally homogeneous populations. ${ }^{1}$ Predictors for PTE include traumatic brain injury (TBI) severity, presence of intracranial bleeding and early post-traumatic seizures (PTS), ${ }^{2}$ although, in 1 study, ${ }^{3}$ the occurrence of early seizures was not an independent risk factor in multivariate analysis. Previous reports have showed that age, neuroimaging results and treatment methods are independent risk factors of PTE, ${ }^{4}$ but others describe that age has not resulted in a significant difference in the probability of experiencing late PTS. ${ }^{5}$ One study showed an increased risk of PTE with increased TBI severity, despite different severity definitions. ${ }^{6}$ Thus, the risk factors for PTE after severe TBI should be further investigated. There is also evidence 
that the risk of PTE may depend on the type of the PTS, with focal or partial seizures tending to recur more often than generalized seizures, ${ }^{7,8}$ whereas Haltiner et $\mathrm{al}^{9}$ did not find a significant predictive relationship between the type of the first late seizure and the probability of seizure recurrence. Nevertheless, it would be preferable to know more about the risk factors, including type of seizures for PTE because such information has important treatment implications.

To clarify these questions, the purpose of this research was to analyze risk factors, including type of seizures for PTE in individuals with at least 1 seizure after severe TBI and provide clues for intervention.

\section{Subjects and methods Subjects}

This is a retrospective follow-up study. The study involved at least 1 PTS patient who had been treated at Liao Cheng People's Hospital between March 2011 and June 2015. The follow-up ended in July 2016. The median duration of follow-up was 86 months from the time of injury and 68 months from the time of the first seizure.

Medical records were retrospectively examined to detect patients who were admitted with severe head injuries (loss of consciousness or amnesia for $>24$ hours, subdural hematoma, or brain contusion ${ }^{10}$ ). In accordance with the standard diagnosis for neurological surgery, a TBI patient was defined as a patient discharged from the hospital with a primary diagnosis of trauma to the head. For inclusion into the study, the following criteria were used: complete medical records and treatment information. Follow-up investigation was conducted via telephone to contactable patients with complete information. The patients with at least 1 seizure after severe TBI went to our hospital for treatment. We asked patients about seizures after the injury, and in case of seizures, we pursued medical notes for confirmation of the diagnosis and recorded the date of the first fit. The time for the onset of seizures during the initial hospitalization had been recorded, which made it possible to calculate the interval from injury to the appearance of seizures. The follow-up survey contained data about the general condition of the patients, the time from injury to the onset of seizures, the type of seizures, the frequency of seizures, the treatment condition, age and the side of injury, and so on. The type of seizures was defined as the most frequent seizures after severe TBI within 12 months.

Individuals were excluded if they were pregnant, had a seizure history before the current TBI, had undergone previous neurosurgery involving dural penetration, or experienced
non-TBI neurologic diagnoses such as brain tumors, stroke, or diabetic coma, and hemisphere of injury. Type of seizures was divided into 3 groups: simple partial seizures (SPS), complex partial seizures (CPS), and complex partial seizures secondary generalized (CPS-G). The patients who could not be placed in any of the 3 groups were excluded from this analysis.

All patients acceded to cranial computerized tomography (CT) scan, and some acceded to brain magnetic resonance imaging (MRI) examination. Side of injury was evaluated based on cranial CT scan or brain MRI examination. Written informed consent for participation was obtained from subjects or their next of kin.

\section{PTS and PTE diagnoses}

Epileptic seizures and epilepsy were diagnosed on the basis of the 1981 and 1989 Classifications of Epileptic Seizures and Epilepsies and Epileptic Syndromes of the International League Against Epilepsy. To be eligible, subjects were required to have obvious trauma history before seizure. PTS was defined as at least 1 seizure after head injury. PTS was classified into early-stage seizures and late-stage seizures according to the time of seizure onset, as follows: early-stage seizures happen within 1 week after trauma; and late-stage seizures occur $>1$ week after trauma. PTE was diagnosed only when 2 or more seizures occurred without acute provocation after the acute phase of injury recovery had passed. ${ }^{4}$ Unprovoked seizures are defined as those that occur more than a week after the head injury. ${ }^{11}$ All cases of PTE were diagnosed by neurologists after a review of the medical charts and follow-up interview data.

\section{Electroencephalogram (EEG) examination}

All the available inpatient 24 hour video-EEG-electrocardiogram (ECG) data for each PTS subject were reviewed. Digital EEG was recorded with a common reference at a sampling frequency of $256 \mathrm{~Hz}$. The EEG data were obtained by using the international standard 10-20 system electrode placement and sphenoidal electrodes.

\section{Statistical analysis}

A database was created in Excel, and statistical analysis was performed using SPSS 17.0 (SPSS Inc., Chicago, IL, USA). The incidence rates are expressed in percentile. The KaplanMeier curves and $\log$-rank $P$-values were used to analyze the probability of PTE by different variables. Cox regression analysis was used to explore relationships between various factors and survival endpoints. The endpoint of the study was 
defined as "PTE" during follow-up. Time to event (seizures) in months, and number of months from injury to follow-up, were used to compute the time variable. Relative risk and 95\% CI for PTE were estimated from regression coefficients. Predictive variables were age at the injury, side of the injury, sex, the time between brain injury and the onset of seizures, type of seizures, and the treatment condition. A significant difference was indicated by $P<0.05$.

\section{Results}

\section{Demographic information}

A total of 79 cases with at least 1 seizure after TBI were admitted to our hospital from March 2011 to June 2015. Among them, 1 case had a history of epilepsy before TBI, 2 cases of injury hemisphere cannot be defined, 3 cases were rigidity and spasm seizures, and 2 cases of the TBI severity were mild and 2 cases were moderate. We attempted to contact the 69 patients by telephone. We were unable to contact 1 patient because the patient had changed the phone number. Among the 68 successfully followed-up patients, 58 were male, and 10 were female. The age at the time of injury ranged from 0.7 to 79 years old, with a mean age of $34.00 \pm 15.96$. The time of the first seizure occurred 1 day to 14 years after brain injury.

\section{PTE diagnoses}

A total of 54 cases were defined as PTE, occurring from 10 days to 179 months after the brain injury, at an incidence rate of $79.4 \%$. Among them, 48 were male and 6 were female; the age ranged from 0.7 to 67 years old. A total of $19(35.2 \%)$ cases had been defined as PTE within the first 6 months after the trauma, 17 cases $(31.5 \%)$ had been defined as PTE within 7-12 months, 8 cases (14.8\%) had been defined as PTE within 13-24 months, 2 cases (3.7\%) had been defined as PTE within 25-36 months, and 8 cases (14.8\%) had been defined as PTE within 37-179 months. Seven cases with PTE had SPS, 9 cases had CPS, and 38 cases had CPS-G. Thirty-two cases had seizure frequencies $<10$ times, 16 cases had seizure frequencies within 11-17 times, and 6 cases had seizure frequencies within 30-200 times within 12 months of the first seizure.

\section{Electroencephalogram (EEG) examination}

All the 54 PTE patients underwent a video-EEG-ECG examination during their hospitalization or on a subsequent visit. All video-EEG-ECG data were reviewed. One case showed normal EEG during interictal, 21 cases showed epileptiform discharges (12 with one-sided discharge, 9 with double-sided discharge), 26 cases showed epileptiform discharges and local cerebral slow wave, and 6 cases had local cerebral slow wave. Abnormal rate of EEG monitoring was $\sim 98.1 \%$.

\section{Characteristics of PTS and probability of PTE}

Of PTS cases, the male-to-female ratio in the study sample was 85.3 to 14.7 . The age of half of the injury subjects was from 8 months to 34 years, and another half was between the age of 35-79 years. The time within 6 months between injury and onset of seizure increased the probability of developing PTE compared with those $>6$ months ([95\% CI, 2.45-7.60] vs [95\% CI, 13.32-46.68], $P<0.01$, respectively [Figure 1; Table 1]). The results showed that the performance of single external puncture drainage or 2 or more surgeries increased the probability of developing PTE compared with those conservative treatment ([95\% CI, 7.15-12.85] vs [95\% CI, 16.74-43.26], $P<0.05$, respectively [Figure 2; Table 1]). The probability of developing PTE was highest for SPS (100\%), followed by CPS (81.8\%), and CPS-G (76.0\%) as indicated in Figure 3 and Table 1. Significant differences between these probabilities were found by using the Log Rank test $(P=0.002)$. In the CPS and CPS-G seizures, no increased probabilities of developing PTE were shown, and the SPS had an obvious statistical significance compared with CPS or CPS-G between these

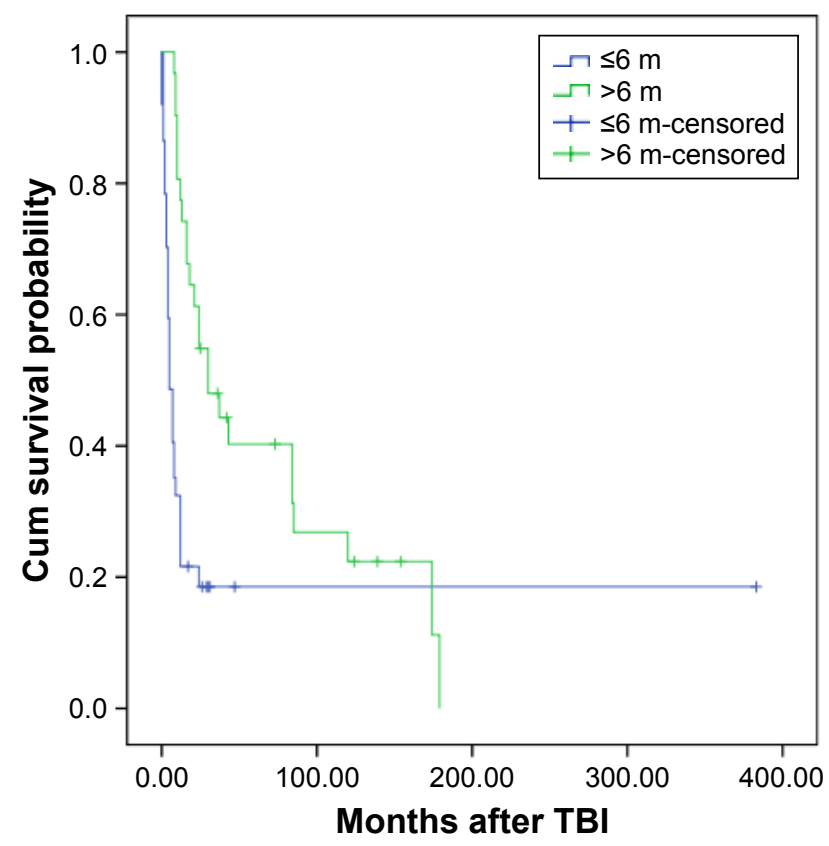

Figure I Kaplan-Meier product limits estimates for probability of survival by the time from injury to onset of seizure.

Abbreviations: m, months; $\mathrm{TBI}$, traumatic brain injury. 
Table I Characteristics of PTS and probability of PTE

\begin{tabular}{|c|c|c|c|}
\hline Variable & $\frac{\text { PTS cases }}{\mathrm{n}(\%)}$ & $\begin{array}{l}\text { PTE cases } \\
\mathrm{n}(\%)\end{array}$ & $\frac{\text { Log rank }}{P \text {-value }}$ \\
\hline \multicolumn{4}{|l|}{ Sex } \\
\hline Male & $58(85.3)$ & $48(82.8)$ & $\chi^{2}=0.444$ \\
\hline Female & $10(14.7)$ & $6(60)$ & $P=0.505$ \\
\hline \multicolumn{4}{|l|}{ Age at injury, years } \\
\hline$\leq 34$ & $34(50.0)$ & $25(73.5)$ & $\chi^{2}=3.598$ \\
\hline$>34$ & $34(50.0)$ & $29(85.3)$ & $P=0.058$ \\
\hline \multicolumn{4}{|c|}{ Time between injury and onset of seizure, months } \\
\hline$\leq 6$ & $37(54.4)$ & $30(81.1)$ & $\chi^{2}=11.697$ \\
\hline$>6$ & $31(45.6)$ & $24(80.0)$ & $P=0.001$ \\
\hline \multicolumn{4}{|l|}{ Treatment method } \\
\hline Conservative treatment & $15(22.1)$ & $8(53.3)$ & $\chi^{2}=4.976$ \\
\hline Surgical treatment & $53(77.9)$ & $46(86.8)$ & $P=0.026$ \\
\hline \multicolumn{4}{|l|}{ Side } \\
\hline Left & $19(27.9)$ & $16(84.2)$ & $\chi^{2}=3.745$ \\
\hline Right & $24(35.3)$ & $20(83.3)$ & $P=0.154$ \\
\hline Bilateral & $25(36.8)$ & $18(72)$ & \\
\hline \multicolumn{4}{|l|}{ Seizure type } \\
\hline SPS & $7(10.3)$ & $7(100)$ & $\chi^{2}=12.805$ \\
\hline CPS & II (I6.2) & $9(81.8)$ & $P=0.002$ \\
\hline CPS-G & $50(73.5)$ & $38(76.0)$ & \\
\hline \multicolumn{4}{|l|}{ Seizure type } \\
\hline CPS & II (I8.0) & $9(81.8)$ & $\chi^{2}=2.384$ \\
\hline CPS-G & $50(82.0)$ & $38(76.0)$ & $P=0.123$ \\
\hline \multicolumn{4}{|l|}{ Seizure type } \\
\hline SPS & $7(10.3)$ & $7(100.0)$ & $\chi^{2}=10.538$ \\
\hline CPS or CPS-G & 61 (89.7) & $47(77.0)$ & $P=0.001$ \\
\hline
\end{tabular}

Abbreviations: CPS, complex partial seizures; CPS-G, complex partial seizures secondary generalized; PTE, post-traumatic epilepsy; PTS, post-traumatic seizures; SPS, simple partial seizures.

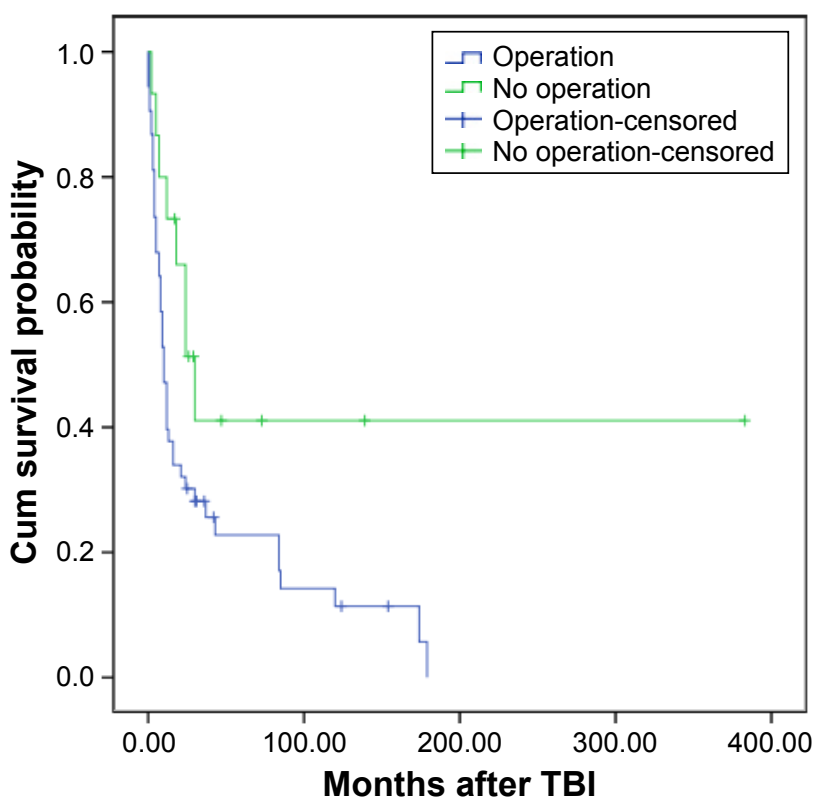

Figure 2 Kaplan-Meier product limits estimates for probability of survival by treatment methods.

Abbreviation: $\mathrm{TBI}$, traumatic brain injury.

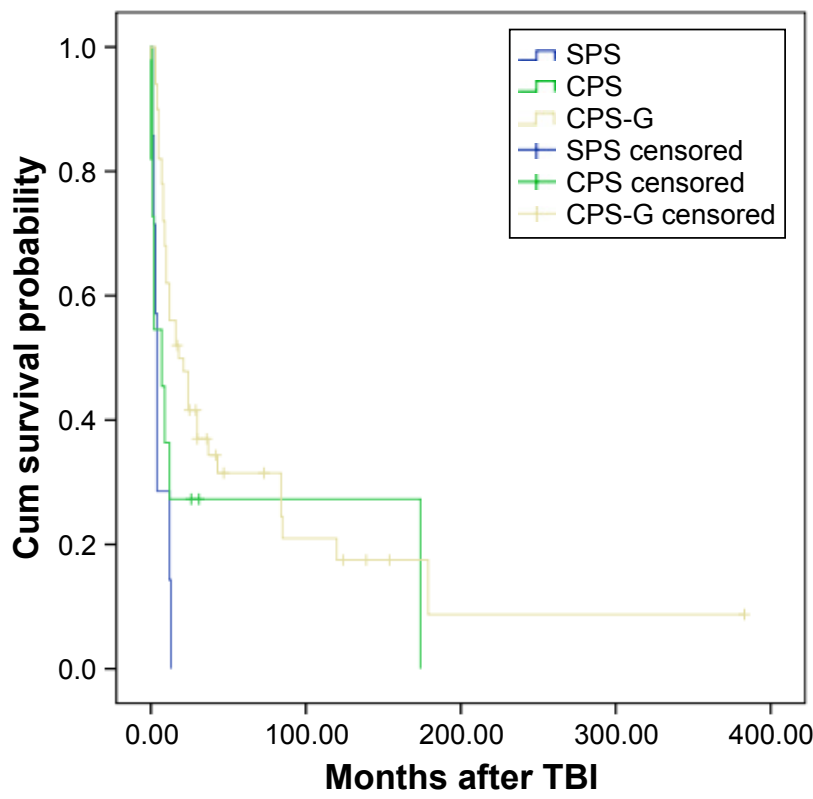

Figure 3 Kaplan-Meier product limits estimates for probability of survival by the type of seizures.

Abbreviations: CPS, complex partial seizures; CPS-G, complex partial seizures secondary generalized; SPS, simple partial seizures; TBI, traumatic brain injury.

probabilities (Figure 3; Table 1). Separate survival analyses for each of the 3 variables sex, age at the time of injury, and side of injury were conducted. None of these 3 factors resulted in a significant difference in the probability of experiencing PTE (Table 1).

Variables, including age, sex, time between injury and onset of seizure, treatment method, side, and seizure type were entered into a Cox regression model, to identify the predictors of PTE. We found that the independent predictive factors for the probability of developing PTE were the time within 6 months between injury and onset of seizure compared with those $>6$ months, the performance of single external puncture drainage or 2 or more surgeries compared with those conservative treatment, and the SPS compared with CPS or CPS-G (Table 2). In particular, the risk of developing PTE for patients aged $>34$ years at the time of injury was 2.55 times greater than for those aged $\leq 34$ years. In

Table 2 Results of Cox proportional hazard model for PTE outcome in 68 patients with at least I seizure after TBI

\begin{tabular}{llll}
\hline Variables & Relative risk & $\mathbf{9 5 \% ~ C l}$ & $\boldsymbol{P}$-value \\
\hline$>34$ years old & 2.547 & $1.402-4.629$ & 0.002 \\
Time between injury and & 0.175 & $0.089-0.346$ & 0.000 \\
onset of seizure (months) & & & \\
Treatment method & 0.256 & $0.113-0.581$ & 0.001 \\
Seizure type & 0.277 & $0.117-0.659$ & 0.004 \\
\hline
\end{tabular}

Abbreviations: PTE, post-traumatic epilepsy; TBI, traumatic brain injury. 
addition, sex and side were not predictive for the probability of developing PTE on Cox regression analysis.

\section{Discussion}

PTS and PTE are complications from TBI. Among the various risk factors of PTE after TBI, the severity of TBI plays an important role as one of the independent risk factors. Variations in estimating the incidence of PTE are due to the differing definitions used for PTS and $\mathrm{PTE}^{1}$ and varying lengths of follow-up in studies. Previous study showed that 21 (23\%) individuals had experienced PTE in samples of only severe head injuries. ${ }^{12}$ A study in China found $26 \%$ of hospitalized TBI cases with early PTS progressed to unprovoked seizures. ${ }^{13}$ Published report shows a PTE incidence rate of $5.0 \%$ during the first 3 years after trauma in 2,826 TBI patients, with $66.0 \%$ occurring within the first 6 months after the injury and $76.9 \%$ within the first 12 months after TBI. ${ }^{4}$ However, there are few studies exploring the prevalence and risk factors of PTE in samples of severe injuries and at least 1 seizure.

In our report, of the 68 PTS patients, 54 cases (79.4\%) were diagnosed as presenting with PTE, occurring from 10 days to 179 months after TBI. Nineteen out of 54 cases were $(35.2 \%)$ presented within 6 months after the TBI, and $36 / 54(66.6 \%)$ presented within the first 12 months after the severe TBI. This result is consistent with those of other similar report, which indicated that most seizures occur within 1 year after injury, although they can also manifest several years after the trauma. ${ }^{1,4}$

Some researchers reported that the occurrence of PTS is not an obvious risk factor of PTE.${ }^{14}$ Nevertheless, our study shows that individuals who experienced both early and late post-traumatic seizure, PTE was evident within 6 months post injury, which had been reported previously. ${ }^{5}$ These results are similar to those reported by most investigators that the risk for PTE after TBI is initially high and decreases over time., ${ }^{3,15,16}$

In our report, we observed that the performance of single external puncture drainage or 2 or more surgeries increased the probability of developing PTE compared with those conservative treatment, as in previous studies. ${ }^{4,5}$ Surgical treatment often involves a large craniectomy and enlargement of the size of the intradural compartment with a patch graft, both of which allow the brain to swell outside of the cranium. The obvious conclusion is that these individuals have quantitatively more brain injury, either multifocal or larger individual foci, which may become epileptogenic over time. ${ }^{5}$

Zhao et $\mathrm{al}^{4}$ noted that several types of seizures can occur after TBI, with partial seizures as the major manifestation.
The same author stated that all 141 patients had partial seizures with or without secondary generalized seizures. Our results are similar to their reported findings. However, previous studies noted that in $67 \%$ of individuals with late PTS, the seizure was generalized or focal with secondary generalization. ${ }^{5,9}$ One of the possible explanations is that partial seizure, such as SPS, may manifest with subtle sensory alterations that can be mistaken for catatonia, or mood alterations associated with conditions such as post-traumatic stress disorder. The presentation of a SPS could be featureless to the untrained eye and might elude detection by a clinician who is unfamiliar with this condition before diagnosis of PTE.

In the present study, the most significant findings on risk factors for PTE have come from the type of seizures follow-up within 12 months after the severe TBI. This is an issue on which previous investigations have been inconclusive. The semiology of PTS may change (or become more apparent) over time,${ }^{17}$ and thus, the seizure type that was initially documented may not always provide a reliable basis for predicting risk of recurrence. ${ }^{9}$ By contrast, there is also evidence that the risk of recurrence may depend on the type of the PTS, with focal or partial seizures tending to recur more often than generalized seizures. ${ }^{78}$ Compared with previous studies in type of seizures, other studies revealed that no difference in seizure prognosis as a function of the time of initial onset. ${ }^{8,18}$ However, no study showeds which particular seizure type was more likely to develop PTE. At least in our study, we found that the type of seizures may be independent risk factors of PTE. In our report, the probability of developing PTE was highest for SPS (100\%), followed by CPS (81.8\%), and CPS-G (76.0\%) as indicated in Figure 3 and Table 1. Significant differences between these probabilities were found by using the Log Rank test ( $P=0.002)$, and the SPS had an obvious statistical significance compared with CPS or CPS-G between these probabilities (Table 1). The differences of these probabilities remain unclear, possibly due to multiple reasons. The most possible explanation is that as Jennett ${ }^{19}$ pointed out, when seizures are initially classified as being nonfocal, further monitoring of subsequent attacks often yields evidence suggesting a focal origin for the seizures. In addition, the result may be affected by the definition of seizure types in this report. Further research on this topic is necessary.

Age is an independent risk factor that affects PTE. Frey ${ }^{1}$ reported that age $>65$ years at the time of injury is a significant risk factor for the development of PTE. In our study, the age was positively correlated with the risk of PTE after TBI. The results show that independent predictive 
factors for the probability of developing PTE are observed in patients $>34$ years of age using Cox regression analysis. Although age was not predictive for the probability of developing PTE on separate survival analyses, we still believe that these factors contribute to the increase in the incidence of PTE among older individuals, given that older patients are less flexible in terms of movement and have systematically reduced physical functions. This observation is consistent with previously reported findings. ${ }^{4}$ There was no difference in the risk factors of PTE by sex, as other studies have shown. ${ }^{20}$ In addition, side was not predictive for the probability of developing PTE on separate survival analyses for the variable.

\section{Limitations}

This study had certain limitations. The current study is limited by the biases inherent to the retrospective nature of its design. In future studies, the scope of sampling must be expanded through a multi-centered investigation. The risk factor of PTE, including type of seizures, must be further investigated to provide patients with an advanced clinical diagnosis and treatment.

\section{Conclusion}

In conclusion, our results emphasize the importance of screening data on seizures for SPS in patients aged $>34$ years at the time of injury, surgical treatment and within 6 months from injury to onset of seizure. The findings of our study suggest that SPS in patients aged $>34$ years at the time of injury, surgical treatment and onset of seizures occurring within 6 months after injury are independent risk factors of PTE.

\section{Ethics approval and consent to participate}

This study was conducted according to the Helsinki Declaration and approved by the ethics committee at the Beijing Tiantan Hospital. Written informed consent was obtained from every participant included in this study.

\section{Acknowledgments}

The authors would like to thank all respondents of the study and everyone who helped in carrying out this study.

\section{Disclosure}

The authors report no conflicts of interest in this work.

\section{References}

1. Frey LC. Epidemiology of posttraumatic epilepsy: a critical review. Epilepsia. 2003;44(Suppl 10):11-17.

2. Verellen RM, Cavazos JE. Post-traumatic epilepsy: an overview. Therapy. 2010;7(5):527-531.

3. Annegers JF, Hauser WA, Coan SP, Rocca WA. A population-based study of seizures after traumatic brain injuries. N Engl J Med. 1998; 338(1):20-24.

4. Zhao Y, Wu H, Wang X, Li J, Zhang S. Clinical epidemiology of posttraumatic epilepsy in a group of Chinese patients. Seizure. 2012; 21(5):322-326.

5. Englander J, Bushnik T, Duong TT, et al. Analyzing risk factors for late posttraumatic seizures: a prospective, multicenter investigation. Arch Phys Med Rehabil. 2003;84(3):365-373.

6. Ferguson PL, Smith GM, Wannamaker BB, Thurman DJ, Pickelsimer EE, Selassie AW. A population-based study of risk of epilepsy after hospitalization for traumatic brain injury. Epilepsia. 2010;51(5):891-898.

7. Weiss GH, Caveness WF. Prognostic factors in the persistence of posttraumatic epilepsy. J Neurosurg. 1972;37(2):164-169.

8. Salazar AM, Jabbari B, Vance SC, Grafman J, Amin D, Dillon JD. Epilepsy after penetrating head injury. I. Clinical correlates: a report of the Vietnam Head Injury Study. Neurology. 1985;35(10):1406-1414.

9. Haltiner AM, Temkin NR, Dikmen SS. Risk of seizure recurrence after the first late posttraumatic seizure. Arch Phys Med Rehabil. 1997; 78(8):835-840.

10. Annegers JF, Grabow JD, Groover RV, Laws ER Jr, Elveback LR, Kurland LT. Seizures after head trauma: a population study. Neurology. 1980;30(7 Pt 1):683-689.

11. Chen JW, Ruff RL, Eavey R, Wasterlain CG. Posttraumatic epilepsy and treatment. $J$ Rehabil Res Dev. 2009;46(6):685-696.

12. Skandsen T, Ivar Lund T, Fredriksli O, Vik A. Global outcome, productivity and epilepsy 3-8 years after severe head injury. The impact of injury severity. Clin Rehabil. 2008;22(7):653-662.

13. Wang HC, Chang WN, Chang HW, et al. Factors predictive of outcome in posttraumatic seizures. J Trauma. 2008;64(4):883-888.

14. Christensen J, Pedersen MG, Pedersen CB, Sidenius P, Olsen J, Vestergaard $\mathrm{M}$. Long-term risk of epilepsy after traumatic brain injury in children and young adults: a population-based cohort study. Lancet. 2009;373(9669):1105-1110.

15. Weiss GH, Feeney DM, Caveness WF, et al. Prognostic factors for the occurrence of posttraumatic epilepsy. Arch Neurol. 1983;40(1):7-10.

16. Temkin NR. Risk factors for posttraumatic seizures in adults. Epilepsia. 2003;44(Suppl 10):18-20.

17. Caveness WF, Meirowsky AM, Rish BL, et al. The nature of posttraumatic epilepsy. J Neurosurg. 1979;50(5):545-553.

18. Caveness WF. Onset and cessation of fits following craniocerebral Trauma. J Neurosurg. 1963;20:570-583.

19. Jennett B. Epilepsy After Non-Missile Head Injuries. Year Book Medical Publishers Inc ed. Chicago: Year Book Medical Publishers Inc; 1975.

20. Angeleri F, Majkowski J, Cacchio G, et al. Posttraumatic epilepsy risk factors: one-year prospective study after head injury. Epilepsia. 1999;40(9):1222-1230.
Neuropsychiatric Disease and Treatment

\section{Publish your work in this journal}

Neuropsychiatric Disease and Treatment is an international, peerreviewed journal of clinical therapeutics and pharmacology focusing on concise rapid reporting of clinical or pre-clinical studies on a range of neuropsychiatric and neurological disorders. This journal is indexed on PubMed Central, the 'PsycINFO' database and CAS,

\section{Dovepress}

and is the official journal of The International Neuropsychiatric Association (INA). The manuscript management system is completely online and includes a very quick and fair peer-review system, which is all easy to use. Visit http://www.dovepress.com/testimonials.php to read real quotes from published authors. 